Here $V$ and VI are trivial: IV follows from the bi-determinantal identity

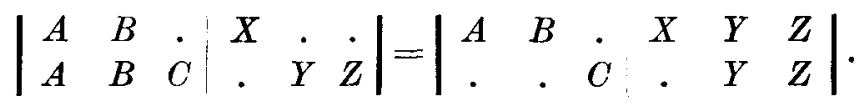

The $\Sigma$ development of such identities at once gives rise to the dual type of Sylvester identities given in 3, p. 95 . With $i=k=2$, from

$$
\Delta=\left|\begin{array}{rrrr}
a & b & c & d \\
\cdot & \cdot & c & d
\end{array}\right| \begin{array}{rrrr}
x & y & z & w \\
\cdot & \cdot & -z & -w
\end{array}|=| \begin{array}{rrrrrrrr}
a & b & \cdot & \cdot & x & y & z & w \\
\cdot & \cdot & c & d & x & y & \cdot & .
\end{array} \mid
$$

we obtain as respective $\Sigma$ expansions

$$
(a b c d \mid x y z w)-(a b \dot{c} \mid x y \bar{z})(\dot{d} \mid \bar{w})+(a b \mid x y)(c d \mid z w)=(a b \mid z w)(c d \mid x y)
$$

which is identity (29) of p. 95 .

\title{
REFERENCES.
}

1. Muir : History of Determinants.

2. Muir and Metzler (New York, 1930), Theory of Determinants.

3. Turnbull: Theory of determinants, matrices and invariants (1928).

4. Turnbull and Aitken : Canonical Matrices (1932).

\section{Pictorial relativity}

By I. M. H. Etherington, University of Edinburgh.

There are some people who find it easier to absorb abstruse theories when encouraged by picturesque analogies. There are also some people, mathematicians, who hold such assistance in austere contempt. Pictorialism, however, is no new thing in expositions of relativity, and I will not apologise for the following attempt to introduce a little more. The object of this note is to suggest ways of visualising some of the metrics which are of importance in the general theory of relativity. For each metric two pictures are supplied; they may be called (A) geometrical and (B) dynamical. The first is got by taking a section of the four-dimensional continuum corresponding to a given metric, and immersing this section in ordinary space. The second shows graphically the magnitude of the 
xxii

gravitational force at any point in the field to which the metric refers. Some interest attaches to a visual comparison of the two figures. The metric of special relativity is pictured by a horizontal plane in both representations.

The figures are purely graphical in character, and nothing more is claimed for them than that they are convenient and appropriate pictures.

Static forms with spherical symmetry.

Consider the metric

$$
d s^{2}=-\gamma(r) d t^{2}+\beta(r) d r^{2}+r^{2} d \theta^{2}+r^{2} \sin ^{2} \theta d \phi^{2},
$$

which includes as special cases a number of important forms considered separately below. To obtain a geometrical picture of the space-time having this metric, since it is static and spherically symmetric, it will be sufficient to consider a section $\phi=0, t=0$. In such a section

where

$$
\begin{aligned}
d s^{2} & =\beta d r^{2}+r^{2} d \theta^{2} \\
& =d r^{2}+r^{2} d \theta^{2}+d z^{2}, \\
d z & =(\beta-1)^{\frac{1}{2}} d r .
\end{aligned}
$$

Hence, interpreting $r, \theta, z$ as cylindrical coordinates in ordinary space, (2) is the metric of the surface of revolution

$$
z=\int(\beta-1)^{\frac{1}{2}} d r
$$

When $\beta(r)$ is known, a meridian section of this surface can be sketched; and this gives our first picture of the metric (1). It is real provided $\beta \geqq 1$, a condition which is satisfied in the examples (i)-(iv) below. It is not a completely adequate representation, since it does not take account of the coefficient of $d t^{2}, g_{44}=-\gamma$; but it is supplemented by the second picture, which depends on this coefficient.

The strength of the gravitational field at any point, as measured by the force on a unit mass there, is given under ordinary conditions $\left(c f .{ }^{1}\right.$ E. 55.4 and 38.5$)$ almost entirely by the Christoffel symbol $\{14,4\}=d(\log \sqrt{ } \gamma) / d r$, and is therefore represented by the gradient of the surface of revolution

$$
z=m^{\prime} \log \sqrt{ } \gamma
$$

${ }^{1}$ References in this form are to equations in Eddington, Mathematical Theory "f Relativity, Cambridge, 1930. 
where $m^{\prime}$ is a constant length. This gives our second picture, which is simply a graph of the approximate analogue of the gravitational potential.

Consider now some special cases, obtained by particularising the functions $\beta(r), \gamma(r)$ in (1).

(i) The Schwarzschild metric (E. 38.8), representing an external spherically symmetric gravitational field. Here

$$
\gamma=1-2 m / r=1 / \beta, \quad r>2 m>0 .
$$

The representative surfaces are:

$$
z=\int_{2 m}^{r}\{2 m /(r-2 m)\}^{\frac{1}{2}} d r=\{8 m(r-2 m)\}^{\frac{1}{2}}
$$

This is half a paraboloid of revolution with meridian sections as shown by the thick lines in Figure 1.

$$
z=\frac{1}{2} m^{\prime} \log (1-2 m / r)=-m m^{\prime} / r-m^{2} m^{\prime} / r^{2} \ldots,
$$

shown by the thick lines in Figure 2. The corresponding model for the Newtonian external spherically symmetric gravitational field is the surface $z=-m m^{\prime} / r$, which of course is practically the same as (4) when $r / m$ is large, but is finite when $r=2 m$.

(ii) Schwarzschild's internal solution of the gravitational equations (E. $72 \cdot 1$, with $a=2 m / a^{3}$ ), representing the internal gravitational field of a sphere of incompressible liquid. Here

$$
\begin{aligned}
\gamma & =\left\{\frac{3}{2}(1-2 m / a)^{\frac{1}{2}}-\frac{1}{2}\left(1-2 m r^{2} / a^{3}\right)\right\}^{2}, \\
\beta^{-1} & =1-2 m r^{2} / a^{3}, \quad r<a, \quad a>9 m / 4>0 .
\end{aligned}
$$

Choosing the constants of integration for continuity with (3) and (4) at $r=a$, the representative surfaces are:

$$
\begin{aligned}
z & =\{8 m(a-2 m)\}^{\frac{1}{2}}-\int_{r}^{a}\left\{2 m r^{2} /\left(a^{3}-2 m r^{2}\right)\right\}^{\frac{1}{2}} d r \\
& =(4 m+a)(a / 2 m-1)^{\frac{1}{2}}-\left(a^{3} / 2 m-r^{2}\right)^{\frac{1}{2}}, \quad r<a .
\end{aligned}
$$

This is a segment of a sphere. The position of the sphere as it joins on to the paraboloid (3), is shown for different values of a (i.e. for varying densities) in Figure 1. The lowest sphere $(a=9 \mathrm{~m} / 4)$ is not admissible physically, since for continuity of the internal pressure $a>9 m / 4$. 
xxiv

$$
z=m^{\prime} \log \left\{\frac{3}{2}(1-2 m / a)^{\frac{1}{2}}-\frac{1}{2}\left(1-2 m r^{2} / a^{3}\right)\right\}, \quad r<a .
$$

The surfaces for various $a$ are shown in section in Figure 2.

(iii) The De Sitter World. (E. 67.33, with $R \sin \chi=r$ and $t$ for $R t$.)

$$
\begin{aligned}
\gamma & =1-r^{2} / R^{2}=1 / \beta, \quad 0<r<k . \\
z & =\int \frac{r d r}{\sqrt{\bar{R}^{2}-r^{2}}}=-\sqrt{R^{2}-r^{2}} . \\
z & =\frac{1}{2} m^{\prime} \log \left(1-r^{2} / R^{2}\right) .
\end{aligned}
$$

The first representative surface is a hemisphere, while the meridian sections of the second have roughly the shape of Figure 3, illustrating the well known fact that a free particle cannot remain at rest in these coordinates except at the origin.

(iv) The gravitational field of a particle (or sphere of incompressible liquid) in a curved (De Sitter) universe. (E. 45·3, with $a=3 / R^{2}$.) For the external field,

$$
\gamma=1-2 m / r-r^{2} / R^{2}=1 / \beta . \quad h<r<k,
$$

where $h, k$ are the positive roots of the cubic equation $\gamma(r)=0, h$ being slightly greater than $2 m$ and $k$ slightly less than $R$. This reduces when $m=0$ to case (iii), when $R \rightarrow \infty$ to case (i).

$$
\begin{aligned}
& z=\int_{h}^{r}\left(\frac{2 m+r^{3} / R^{2}}{r-2 m-r^{3} / R^{2}}\right)^{\frac{1}{2}} d r . \\
& z=\frac{1}{2} m^{\prime} \log \left(1-2 m / r-r^{2} / R^{2}\right) .
\end{aligned}
$$

The rough shapes of the surfaces can be found by straightforward methods of approximation. Completing the surfaces for the internal field by analogy with Figures 1 and 2, we obtain Figures 4 and 5 . The upper half of Figure 4 can be omitted or considered to coincide with the lower half.

(v) The gravitational field of a point electron. (E. 786 et seq.)

$$
\gamma=1-2 m / r+2 a m / r^{2}=1 / \beta,
$$

where $a / m$ is large so that, unlike cases (i)-(iv), $\beta$ and $\gamma$ are positive for all values of $r$. The equations of the representative surfaces are

$$
\begin{aligned}
z & =\sqrt{2 m} \int^{r}\left(\frac{1-a / r}{r\left(1-2 m / r+2 a m / r^{2}\right)}\right)^{\frac{1}{2}} d r \\
z & =\frac{1}{2} m^{\prime} \log \left(1-2 m / r+2 a m / r^{2}\right) .
\end{aligned}
$$


(Figures 6 and 7.) The modification of these surfaces for a non-zero cosmological constant is easily imagined on the analogy of case (iv) as compared with (i) and (ii).

The vacuity in Figure 6, within the ridge of cusps $r=a$, arises in a different way from the vacuities in Figures 1 and 2 for case (i). These arose because the signs of $\beta$ and $\gamma$, and hence the signature of the original $d s^{2}$, changed when $r<2 m$, so that inside this sphere the metric was no longer appropriate to a representation of space-time. But the $d s^{2}$ of the electron field does not change its signature in this way, and in the spatial section which we are considering the form $d s^{2}=\beta d r^{2}+r^{2} d \theta^{2}$ remains positive definite; but when this is expressed in the form $d s^{2}=d r^{2}+r^{2} d \theta^{2} \pm d z^{2}$, it is the sign of $d z^{2}$ which changes. In other words, when we attempt to immerse the spatial section in a euclidean or pseudo-euclidean 3-space, it is the signature of this 3 -space which changes. The vacuity in Figure 6 is thus a defect of the representation: we cannot deduce from it that the sphere $r=a$ is a genuine singularity of the field-unless indeed there be some reason for postulating that "space" must always be capable of immersion in a euclidean (not pseudo-euclidean) space of higher dimensions.

Expanding universes.

Returning to a consideration of the De Sitter world, it is known that its metric (1) (5) can by a transformation of coordinates be put in the form

$d s^{2}=-d t^{2}+R^{2} \cosh ^{2}(t / R)\left(d \zeta^{2}+\sin ^{2} \zeta d \theta^{2}+\sin ^{2} \zeta \sin ^{2} \theta d \phi^{2}\right)$,

(E. $67 \cdot 31$, with $R(\pi / 2-\omega)=i t)$ which exhibits it as one of the series of expanding universes discovered by Friedman. In these coordinates a material particle can remain at rest at any point of space, so the second of our representative surfaces is simply a horizontal plane. To obtain an analogy of the first representation we note that a spatial section $t=$ constant can be regarded as a hypersphere-or, suppressing two spatial dimensions, a circle-of expanding radius

$$
r=R \cosh t / R \text {. }
$$

Consider then the surface of revolution with this equation, generated by rotating a catenary about its directrix. Its transverse section $t=$ constant is a circle which expands as $t$ increases; and this 
$\mathrm{xxvi}$

expanding circle, or an expanding sphere with the same diameter, may be regarded as picturing the metric (7). (See Figure 8.) A similar picture could be given for any of the expanding universes of Friedman' $^{1}$ and Lemaitre ${ }^{2}$.
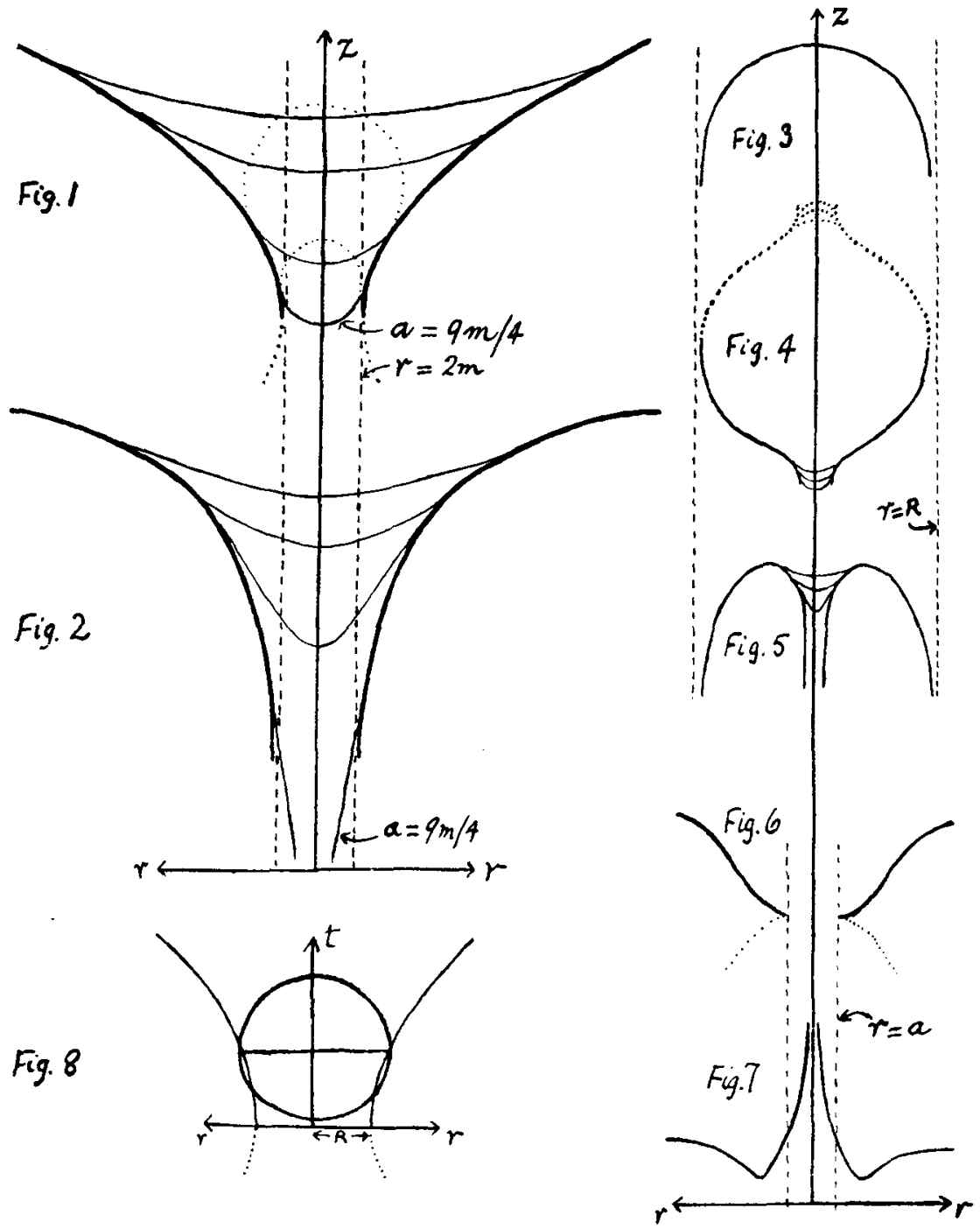

Fig. 8

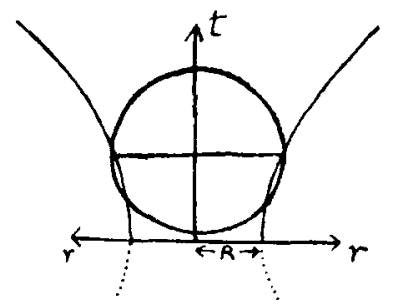

1 Zeit. f. Phys. 10 (19:2), 385.

${ }^{2}$ Monthly Notices R.A.S. 91 (1931), 483. 